\title{
Efficient Routing in Wireless Sensor Network by using Water Cycle Algorithm to Evaluating the Performance of Density Grid Based Clustering
}

\author{
Shradha Yadav, Shalley Bakshi, Manpreet Kaur
}

\begin{abstract}
Wireless communication has an exceptional network known as a wireless sensor network, which consists of many dedicated sensors. They have characteristics of sensing capacity and have the capability to complete a common task. To increase the performance of the network an energy efficient algorithm is needed which enhances the network lifetime and make the system more energy efficient. In this paper, the density grid based clustering is enhanced using the water cycle algorithm. The water cycle algorithm is a nature-inspired optimization algorithm that is used to increase the performance of the network which effects the lifetime of the network. The simulation is done in the Matlab r2015a environment. The proposed methodology has increased the performance of the network when compared with the existing algorithm ABC (artificial bee colony) and its better improvement is shown in parameters like network lifetime, computation time and clusterformed.
\end{abstract}

Keywords: WCA, WSN, optimization

\section{INTRODUCTION}

WSNconsistsofmultiplesensorsthatarespatiallydispersed and assigned to monitor the action in the environment. WSN calculates the conditions in the environment related to temperature, pollution levels, and humidity. The base station gets data when these sensors cooperate and connect with each other and pass their data. Nowadays advanced networks are present having properties of bidirectional and control the activity of sensors. Over the past few years, there is a rapid growth in WSN because of its low power usage battery, wireless communication, its sensor technology and widely used of analog circuit and of its small size of equipment used. They are widely used in applications that contain the operation related to monitoring and detection in the real environment. The network consists of a large number of sensor or hubs of sensor which are closely deployed to perform multifunction activity including sensing and monitoring, processing and communication capabilities, includingtheeventswhicharehappeningintheenvironment.

These nodes have various equipment along with sensors, processors, receiver, and transmitter, power source. The architecture of the sensor node is given in Fig.1.Thesesensor nodescollectivelyperformthefunctionofsendingthesensed

Revised Manuscript Received on July 02, 2019.

Shradha Yadav, Electronics and communication, Chandigarh engineering college, Landran, Mohali, India

Shalley Bakshi, Electronics and communication, Chandigarh engineering college, Landran, Mohali,India.

Manpreet Kaur, Electronics and communication, Chandigarh engineering college, Landran/ Mohali,India datathroughradiowavestobasestationoralsoknownassink mode.

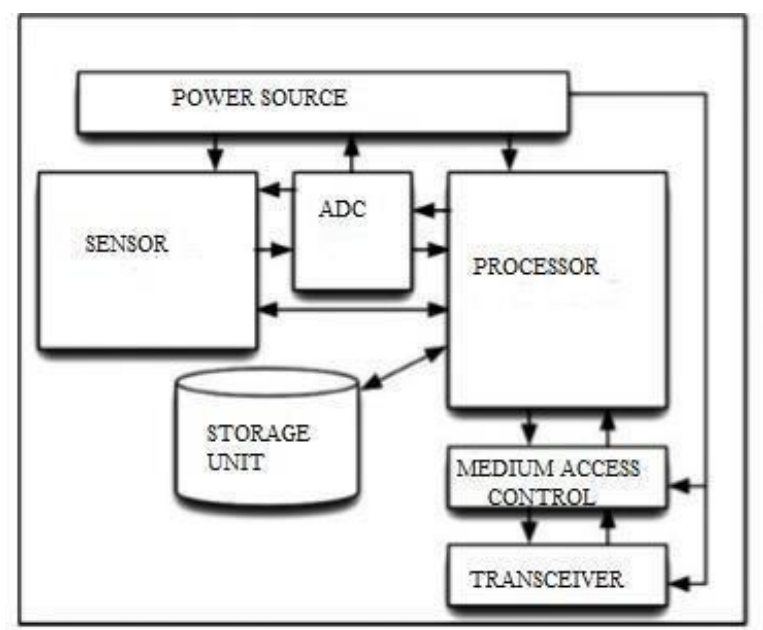

Fig.1 Architecture of sensor node in WSN

The main focuses of the sensor networks are to increase the network lifetime while performing the operation of data communicationandreduceconnectivitydegradationbyusing multiple routing techniques. Due to their ability of sensing, monitoring and detection they are used in a wide range of applications in different fields including health care services, environment (monitoring of any particular habitat, volcanic activity, earthquake), military(enemy detection, monitoring the borders). Nodes that are present in the network are resource constraints because of limited battery supply, their small size processors, so there is a need for an efficient routing protocol to increase their efficiency. These protocols have the ability to discover the routes which make communication faster and efficient. Multiple clustering is developing nowadays which improves the efficiency of the network. These clustering techniques are integrated with differentoptimizationalgorithmwhichhelpsinincreasingthe network lifetime andmake network more efficient. 


\section{A Density grid basedclustering}

By the combination of grid based clustering and density based clustering, the formation of density grid based clustering takes place. In this type of clustering network topology is more stabilized, more fault tolerance ability, maximum network lifetime, and increases the network connectivity. It works on dynamic networks. The processing time calculated in density grid based clustering is quite fast.

\section{LITERATURESURVEY}

2017 Baljinder Singh proposed a methodology on thedensity grid based clustering using $\mathrm{ABC}$ and show the behavior of bees and how they work and pass information[1].2016 Utpal Kumar Paul proposed a novel grid based energy efficient routing algorithm for wireless sensor networks. In which topology is divided into grid shaped on the basis of static cluster protocol, the selection of cluster head is done. Itgives every node an opportunity to become a cluster head [2].2015 ManalAbdullahetalproposedanewclusteringtechniquefor density grid based clustering in WSN. According to this method, the network topology is divided into grids and their classification is done on the basis of highly dense and empty grids respectively to the number of the nodes which are present.Excludingemptygrids, thesegridscombinetoforma cluster, two neighboring low dense grids form an outlier of a cluster. For the selection of cluster head, cluster nodes are classified into an advance node that has less distance to the base station when compare the distance of other nodes to the base station. Afterward, the cluster head will be selected on the basis ofthe highest energy. According to this method, the most suitable grid size which has best results increases the lifetime of the nodes in the network area [3].2014 D Kumar proposedtwoclusteringprotocol(MEECP)multi-hopvitality efficientgroupingconventionandsinglehopvitalityefficient bunching protocol (SEECP) [4].2014 Stefanos et al explains the equalized cluster head selection routing protocol. For the selection of cluster head in the network using the Gaussian elimination algorithm. For the transmission of data from source to base station the routing which is used is multi-hop routing [5].2012 J Peng et al proposed an algorithm that has its origin from LEACH and nodes adaptive schedule was designed for better latency, energy efficiency network [6].2011 Ashok .et.al. Explain a protocol that is locationbased, which works on cluster head selection, routing of data and make network energy efficient which promotes network lifetime. With the use of clustering in the region of where the operation of sensing is required it helps in cluster formation which is of balanced size and less number of transmissions and receiving of operations is required. For ensuring that in the node, energy dissipation is balanced by clusterheadrotation.Inthismethod, therotationofthecluster head becomes more efficient if parameters like distance, energy density are given importance rather than focusing on residual energy[7].2010Nan et. Al in this paper ADAM (adaptive data aggregation mechanism) is adopted to achieve thehighoptimalaggregation,clusterheadverifyeverypacket received from its surrounding nodes on the basis ofstatically character it contains. It transforms the data into shorter blocks. To reduce the level of redundancy in the internode. ADAM has an efficient compression ratio and increases the lifetime of the networks [8].2010 Liu Xiolong proposed a bacterial foraging swarm optimization technique [9].2009 Xiarong et al, works on the basis of the location of a node, its efficiency in communication and its network connection it uses static cluster formation. The selection of cluster head is insuchawaythatthelifetime ofclusterheadincreasesituses optimal scheduling. The work of the cluster head is uses of minimal energy routing technique and collection of data aggregation which is periodical in nature. By using the above method the network lifetime increases along with effective utilization of energy in a manner that is balanced in nature [10]. 2009 Gao et al proposed a protocol known as recluster LEACH protocol is based on the cluster and its node density which is inside the cluster and a mechanism that is based on cluster-baseddatafusion. Thelimitationcreatedbythesingle hop LEACH protocol is overcome in this protocol. To increase, the network lifetime and its energy efficiency multi-hop algorithm are used for cluster head selection [11].2009 Dongkyun et al works on (DAD) duplicate address detectionschemeforeverynodeinthenetwork. Theprotocol mainattentionisonthepassiveschemeofDADwiththegoal in which increase the accuracy of detection of address conflicts. Increase the detection of success ratio and decrease the time required to find these conflicts. Some more information are including location, its sequence number, neighbor knowledge. An increase in accuracy and less time to find conflict addresses and but IP address allocation schemes are also given importance [12]2008Jamping et al works on thealgorithm which is known as time-based cluster head selection to increase the execution of LEACH (TB LEACH).The selection of the cluster head is based on a random number that is assigned to every node in a random time interval. When a comparison is done the number of clusterformationislessintheTB-LEACHiswhencompared to LEACH. The TB LEACH decreases the formation of a number of clusters and also works on simplification for the process of selection of cluster head[13].

2007 Baek et al works on with the help of stochastic

geometric and queuing models how to improve the spatial balance of energy burdens and with the help of using the proactive multipath increase the tradeoff between the energy costs of spreading traffic. Then the comparison is done with thehelpofroutingwhichisknownasshortpathroutingwhich has more initial energy [14]. $2006 \mathrm{Uk}$-Pu Han works on routing protocol which is cluster-based which adds a small slot in a frame; it also provides the facilities of exchange of residualenergymessagesamongclusterhead, nodesandbase station. Theenergystatusisgatheredbytheclusterheadofall nodestowhichtheybelongandsendsthemtothesinkorbase station. After thatthis operation is received by every node

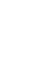


With the help of respective cluster heads they had. For the beginning of the second round, everyone is ready [15].2004 Younis et al explains in the ad hoc sensor network an approach which is used is novel distributed clustering. In this method, the consideration of infrastructure and node capability is not there. The simple assumption of multiple powerlevelnodesispresentinthenetwork. Theselectionfor the procedure of the cluster head is done on the basis of the nodes residual energy, its proximity from its neighbor. It also does data aggregation and it works only for two-level hierarchy[16].

\section{GAPS IN THE LITERATURE SURVEY}

Wireless communication is based on data transfer and its processing ability. The receiving of data at the base station sometimes causes flooding of information. Tosolvetheaboveproblemdataaccumulationmeth odshould be used but this method has not been given much attention.

Theeffectofdatalossduringthefloodingofdataisnotgiven much attention by theresearchers.

With the goal of less data loss and more lifetime, fewer computation times a new technique is used which solves the problem of enhancing the network lifetime and decreases the computation time.

\section{PROPOSEDALGORITHM}

The proposed algorithm is used to improve the network lifetimeaswellasenhancingthedensitygridbasedclustering by using the routing water cycle optimization algorithm. The simulation is done in the Matlab environment (Matlab r2015a). The flowchart of the proposed methodology is shown in Fig.2.

The proposed methodology is done in three phases

A. Initializationphase

B. Procedure for the selection of cluster headphase Routingphase
ISSN: 2278-3075, Volume-8, Issue-9S, July 2019

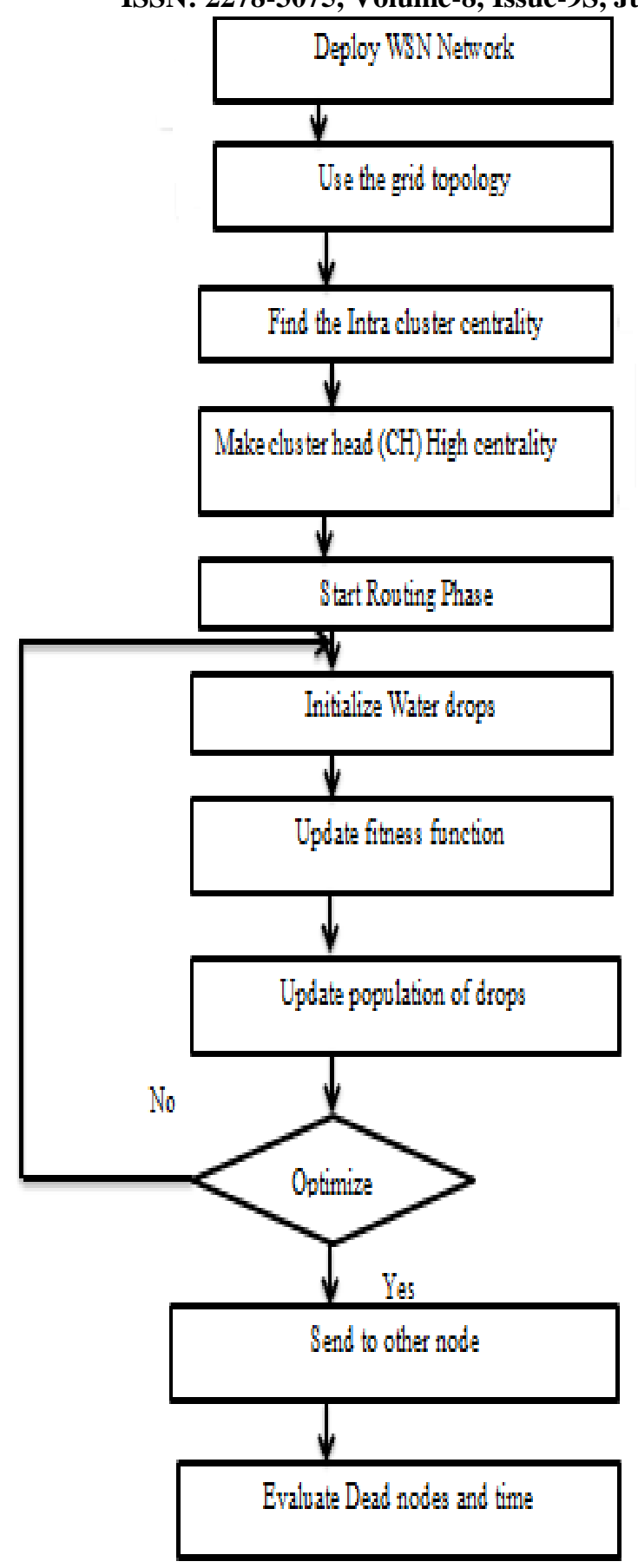




\section{A. Initializationphase}

WSN is deployed in the given area where data or information is collected and send to the base station or sink.

Grid (density grid based) topology is used in the network.

Each grid in a network represents a cluster.

B. Procedure for the selection of cluster head phase Intraclustercentralityiscalculated,tofindout whichnodein the cluster on the terms of the number of its connection it makes with other nodes, how fast they convey informationin the network. The node with high centrality becomes acluster head $(\mathrm{CH})$ in a cluster. The work of the cluster head is the collectionofinformationfromitsnodewhichispresentinits cluster and transfer of this information. There is a restriction oftransferringinformationwithinthenodesthemselves. They can only communicate or send their information to their cluster head which is respectively present in theirclusters.

\section{Routing process}

The routing phase plays an important role in the proposed methodology because it helps in finding the best route for transferring the data to the base station or sink. It helps in increasing the performance of the network by decreasing the usageofbatteryusedbythesensorsandincreasethelifetime, computationtimesofthenetwork.Herewatercyclealgorithm is used for routing the data to the base station or main location. The water cycle imitates the flow of streams and riverstowardstheseaoroceanandwasinspiredbyobserving theprocessofthewatercycle.Intheinitialphaseofthewater cycle algorithm, the initial population of the design variable (number of streams) is the randomly generated after raining process. The best variable or the best stream selected on the basis of the minimum cost function is chosen as the sea. The stream which has value is close to the best variable aschosen as rivers, the remaining variable is considered as streams whichflowintotheriverandsea.Theextentoftheflowofthe river decides the absorption of water from the stream. Hence thevolumeofwaterenteringariver, seavariesfromstreamto stream. The river whose location mostly downhill flows into the sea. All rivers and sea have a specific number of streams which flow into them. The interchange of positions is done between river and stream when compared with its solution given by stream is more optimal when compared with its connectingriver.Asimilarinterchangecanbedoneforariver and a sea. For the exploration phase in the water cycle, the operator used is evaporation. It causes water to evaporate from the sea, rivers, and streams. It basically used for local optima to avoid the situation of premature convergence. Finally, the calculation of distance is done whether stream, a river which has a short distance to the sea. Afterevaporation, condensation of water vapor occurs and rainingprocess repeat and new streams which are formed to specify their new locations, a uniform random search is used [17]. The stepsinvolved in the water cycle algorithm (optimization) are asfollows:

- Generate the initialpopulation.

- Calculate the fitnessfunction.

- Find the best solution and update the fitnessvalue.

- Check the objectivefunction

- Check it optimize ornot

- If optimized then analysis the time and dead node otherwisecheckthecounterisgreaterthan0ornot.If thecountervalueislessthannotconvergethenignore the node duringrouting.

- Else again initialize the value at WCA.

\section{Performancesanalysis}

This paper has implemented the proposed algorithm in the Matlab r2015a software. In part A the existing Artificial Bee Colony (ABC) technique is discussed and in part $\mathrm{B}$ the proposed (improved) water cycle algorithm (WCA) is integrated to enhance density grid based clustering is discussed.

\section{Part A}

The exiting technique artificial bee colony is discussed and the various parameters are shown in the graph. The value obtained by the graph on three parameters which are cluster formation, network lifetime, computation time is represented in table 1 .

$\mathrm{ABC}$ technique which is used for optimization. This technique is based on honey bee foraging behavior and was developed by Dervis Karabogain 2005. The model of ABC consists of

1. Employedbees

2. Onlookersbees

3. Scoutsbees

This technique is a population-based algorithm. The food source location corresponds to a possible solution for the optimization problem and the food source (amount, quality) representstheassociatedsolution.Butthedisadvantageofthe

$\mathrm{ABC}$ technique is it performs only local optimization. To overcome this problem water cycle optimization algorithm is used in proposed work and it performs local and global optimizationboth

Table 1 shows the existing technique (ABC) is on parameters cluster

Formation, network

lifetime, computation time.

Computation time defined as

thelength of timerequiredto 
perform a computation process.Network lifetime defines an operational time of the network in which it is able to perform the dedicated task.

\begin{tabular}{|c|c|c|c|}
\hline \multirow{2}{*}{$\begin{array}{l}\text { No. } \\
\text { of } \\
\text { nodes }\end{array}$} & \multicolumn{3}{|c|}{ Value by ABC( ARTIFICIAL BEE COLONY) } \\
\hline & $\begin{array}{l}\text { Cluster } \\
\text { formed }\end{array}$ & $\begin{array}{l}\text { Network } \\
\text { lifetime }\end{array}$ & Computation time \\
\hline 100 & 211 & 336 & 0.3697 \\
\hline 150 & 210 & 337 & 0.5227 \\
\hline 200 & 211 & 342 & 0.7011 \\
\hline 250 & 198 & 338 & 0.8614 \\
\hline 300 & 189 & 337 & 1.0389 \\
\hline
\end{tabular}

The graph is given by Artificial Bee Colony (ABC) existing technique

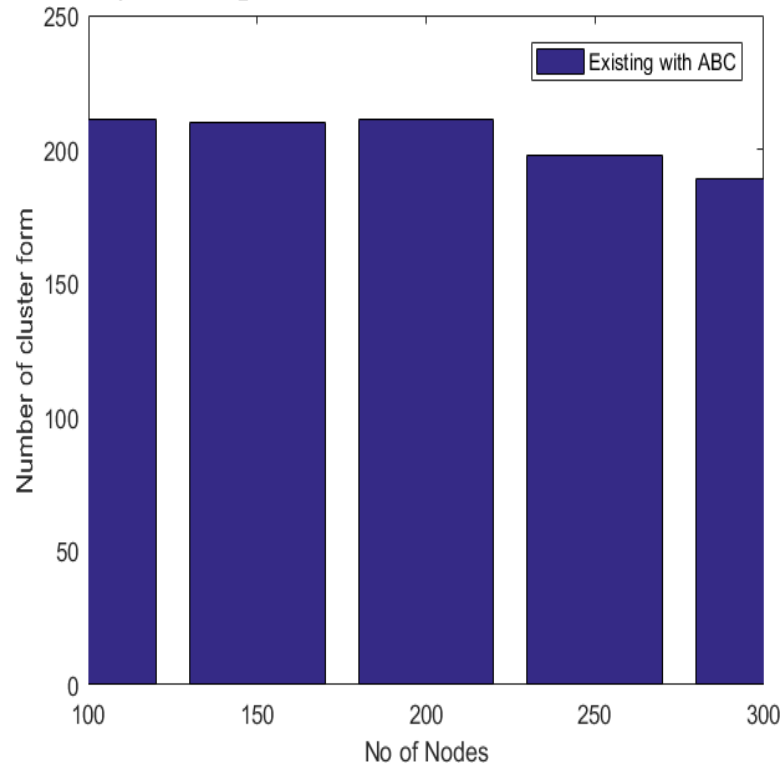

Fig.2 Cluster formed

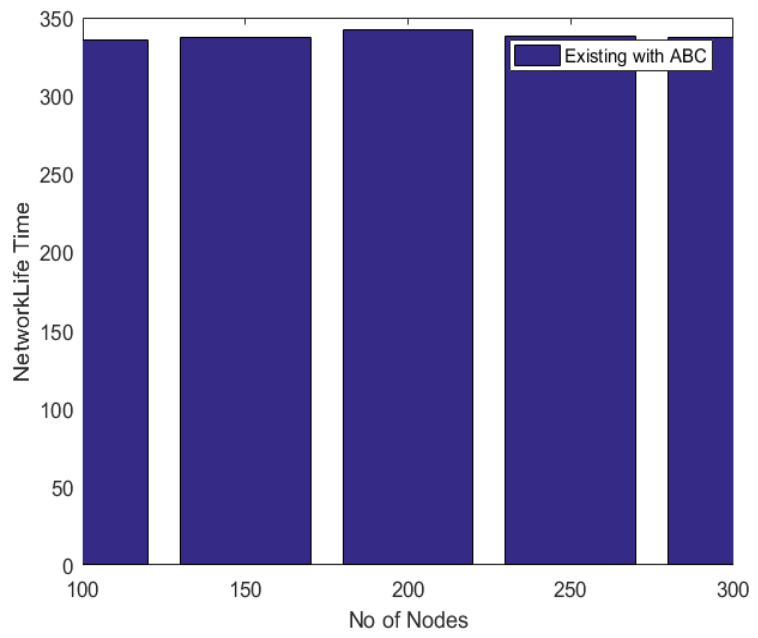

Fig. 3 Network lifetime

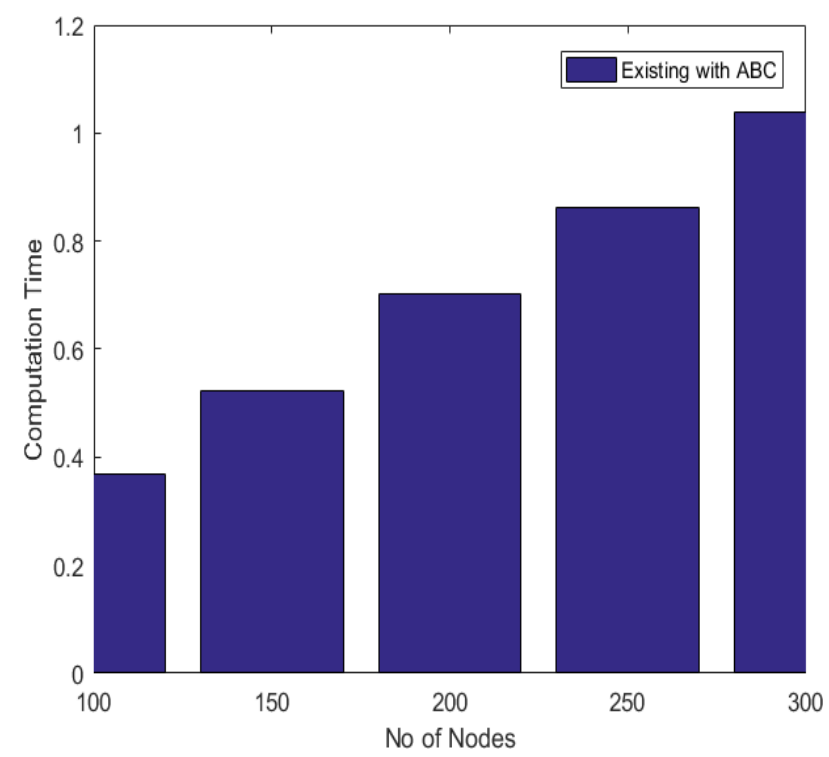

Fig. 4 computation time

\section{Part B}

In $\mathrm{B}$ part of the section, the proposed routing (water cycle algorithm) is integrated to enhance the density grid based clustering.Thisoptimizationalgorithmisusedinthenetwork for routing the data. The graph obtains by the proposed algorithm(WCA)showssignificantimprovementinthevalue which was given by the $\mathrm{ABC}$ technique. The physical and wireless parameters of the proposed methodology are given below in table2 
Efficient routing in wireless sensor network by using water cycle algorithm to evaluating the performance of density grid based clustering

TABLE 2 SETTLING PHYSICAL AND WIRELESS PARAMETERS

\begin{tabular}{|l|l|}
\hline Operation & Energy dissipation \\
\hline Shape of network & Grid/ Square \\
Number of Nodes & $10^{4} \mathrm{~m}^{2}$ \\
Transmitter/ Receiver & $100-300$ \\
Data Aggregation & $\begin{array}{l}\text { Eelec }=\mathrm{E}_{\mathrm{tx}}=\mathrm{E}_{\mathrm{rx}}=50 \\
\mathrm{~nJ} / \mathrm{Bit} \\
\text { Ers }\end{array}$ \\
& $10 \mathrm{PJ} / \mathrm{Bit} / \mu \mathrm{m}{ }^{4}$ \\
& $\begin{array}{l}\mathrm{Emp}=0.0013 \mathrm{PJ} / \mathrm{Bit} \\
\mathrm{m}^{4}\end{array}$ \\
\hline
\end{tabular}

The water cycle algorithm is the best algorithm when compared with other routing algorithms. It is a natureinspired algorithm which is based on the concept of rivers and streams flow in the sea. This algorithm is able to computethemaximumandminimumvalueofthefunction.It works on user-defined fixed variable and it addresses a large number of the optimization problem. It performs local and global optimization. ABC supports only local optimization. Theresultgivenbythewatercyclealgorithmisshownintable 3 .

TABLE 3 VALUES GIVEN BY PROPOSED WATER CYCLE ALGORITHM

\begin{tabular}{|l|l|l|l|}
\hline \multirow{2}{*}{$\begin{array}{l}\text { No. } \\
\text { of } \\
\text { nodes }\end{array}$} & \multicolumn{3}{|c|}{ Value by WCA( water cycle algorithm) } \\
\cline { 2 - 4 } & $\begin{array}{c}\text { Cluster } \\
\text { formed }\end{array}$ & $\begin{array}{c}\text { Network } \\
\text { lifetime }\end{array}$ & Computation time \\
\hline & & & \\
100 & 278 & 340 & 0.2342 \\
150 & 234 & 339 & 0.4532 \\
200 & 267 & 350 & 0.6544 \\
250 & 200 & 345 & 0.7000 \\
300 & 190 & 347 & 0.8979 \\
& & & \\
\hline
\end{tabular}

The graph shown by the water cycle algorithm proposed an algorithm

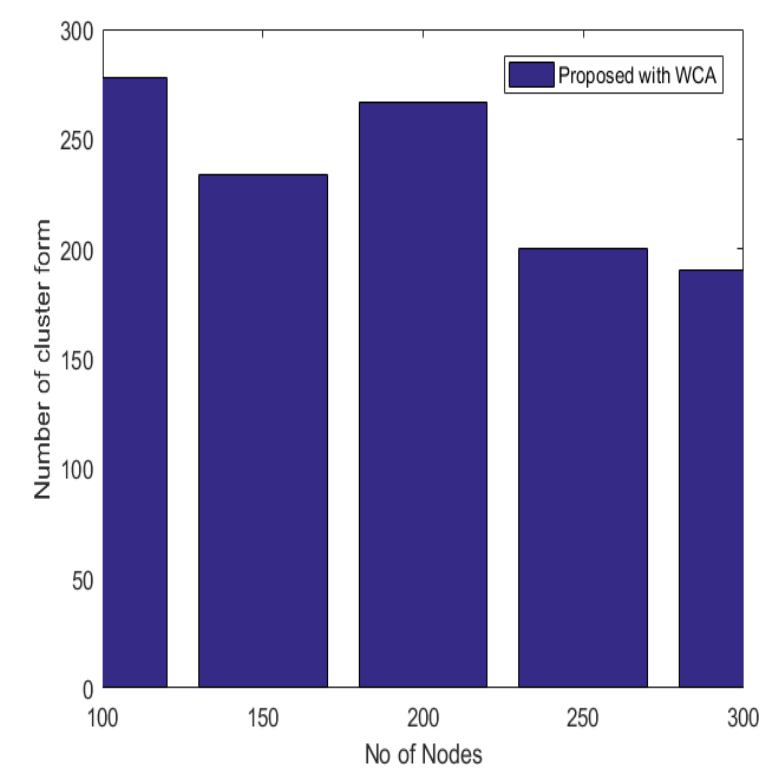

Fig.5 number of cluster form

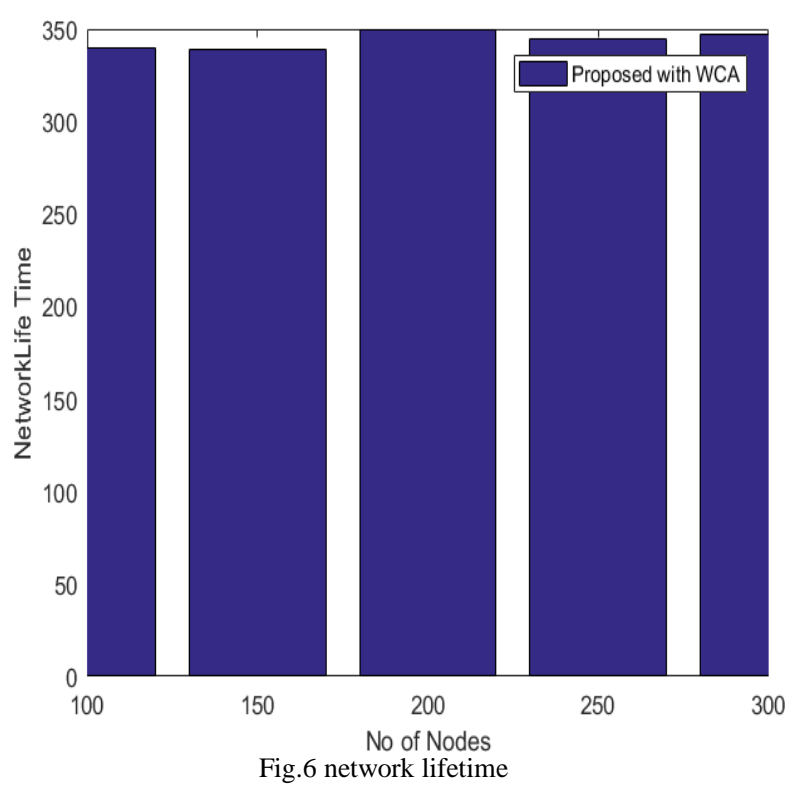




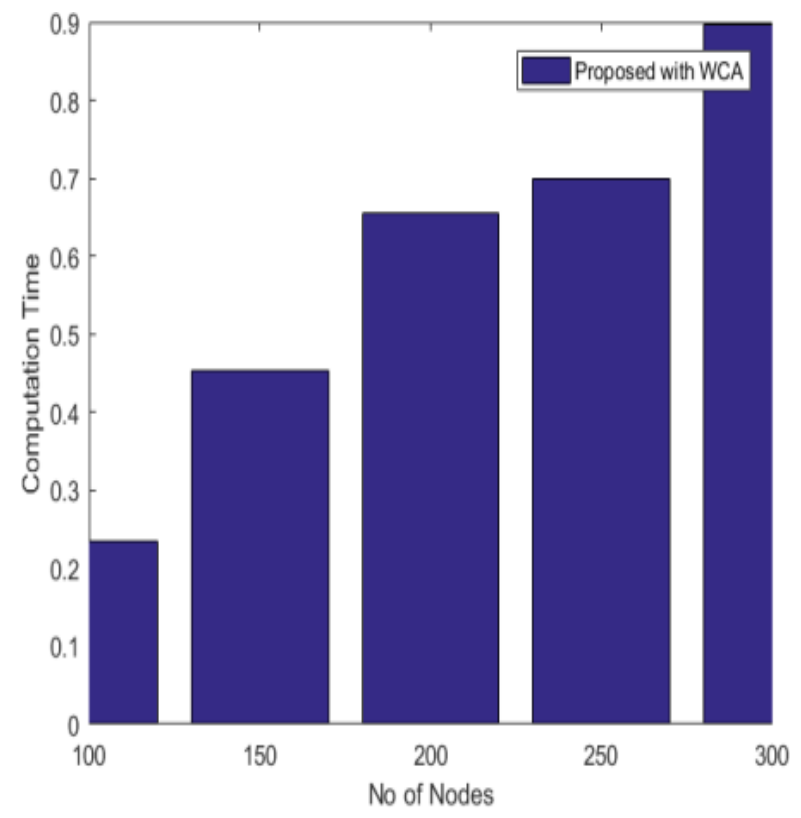

Fig.7 computation time

FromthegraphgivenbyWCAonclusterformation,network

lifetime, computation time it is clear that the proposed algorithm has improved the result by $12.83 \%$ in clusterform, $2 \%$ in network lifetime, $18.8 \%$ in computation time when compared with existing $\mathrm{ABC}$ technique [1]. The simulation done is best on the environment having 300nodes.

\section{CONCLUSION}

The concentration of research work is on developing an efficient routing that optimizes the route from cluster head to thebasestationwhichaffectstheparameterslikecomputation time,networklifetime,clusterformationandwhichimproves system stability and lifetime. The proposed strategy is composed and executed in the Matlab software. The water cycle algorithm is used to perform local and global optimization. This work can be further extended to implementing in such an application where throughput, energy conservation isimportant.

\section{REFERENCES}

[1] Baljinder Singh,"evaluating the performance of density grid based clustering using ABC technique for efficient routing in WSNs" D.O.I:10.1109/CISS.2017.7926099,Conference:2017 ,51 annual conference on information sciences andsystem(CISS)

[2] Utpal Kumar Paul, Sudipta Chattopadhyay, "A Novel Grid based energy efficient Routing Algorithm for Wireless Sensor Network", International Conference on Wireless Communications, Signal Processing and Networking (WiSPNET), IEEE, pp 223-226,2016.

[3] Abdullah, Manal, et al. "Density Grid-Based Clustering for Wireless Sensors Networks." Procedia Computer Science 65 (2015):35-47.

[4] Kumar Dilip "Performance analysis of energy efficient clustering protocols for maximizing lifetime of wireless sensor networks." Wireless Sensor Systems, IET 4, No. 1, pp. 9-16,2014.

[5] Stefanos A. Nikolidakis, Dionisis Kandris, Dimitrios D. Vergados, and Christos Douligeris, (2013)"Energy Efficient Routing in Wireless Sensor Networks Through BalancedClustering,"

[6] Ji, Peng, Yupeng Li, Jingqi Jiang, and Tianbao Wang. "A Clustering Protocol for Data Aggregation in Wireless Sensor Network." In Proceedings of the IEEE International Conference on Control Engineering and Communication Technology, pp. 649-652,2012.

[7] Ashok Kumar and Vinod Kumar, (2011)“Energy Efficient Clustering and Cluster Head Rotation Scheme for Wireless Sensor Networks,"(IJACSA) InternationalJournalofAdvancedComputerScience and Applications, vol. 3 , no. 5

[8] Li, Nan, Shangru Li, and Xiaozhou Fang. "Adaptive data aggregation mechanism based on leach protocol." in International Conference on Advanced Intelligence and Awareness Internet (AIAI 2010), pp. 131134,2010

[9] Liu XiaoLong, Li RongJun, YangPing, "A Bacterial Foraging Global Optimization Algorithm Based On the Particle Swarm Optimization", International Conference on Intelligent Computing and Intelligent Systems, IEEE, pp. 21-27, December2010.

[10] Xiaorong Zhu, Lianfeng Shen, and Tak-Shing Peter Yum, (2009)"Hausdorff Clustering and Minimum Energy Routing for Wireless Sensor Networks," IEEE Transactions on Vehicular Technology, vol. 58, no.2.

[11] Gao Yi, Sun Guiling, Li Weixiang, and Pan Yong, (2009)"ReclusterLEACH: A Recluster Control Based on Density for Wireless Sensor Network," International Conference on Power Electronics and Intelligent TransportationSystem.

[12] Dongkyun Kim, Hong-Jong Jeong, C. K. Toh, and Sutaek Oh, (2009)"Passive Duplicate Address-Detection Schemes for On- Demand Routing Protocols in Mobile Ad Hoc Networks," IEEE Transactions on Vehicular Technology, vol. 58, no.7.

[13] Hu Junping, Jin Yuhui, and Dou Liang, (2008)“A Time-based ClusterHead Selection Algorithm for LEACH,” IEEE Symposium on Computers andCommunications,

[14] S. J. Baek and G. de Veciana, (2007) "Spatial Energy Balancing through Proactive Multipath Routing in Wireless Multihop Networks," IEEE ACM Transactions Networking, vol. 15, no. 1, pp. 93-104,February

[15] Uk-Pyo Han, Sang-Eon Park, Seung-Nam Kim, and Young-Jun Chung, (2006)"An Enhanced Cluster Based Routing Algorithm for Wireless Sensor Networks," IEEE Transactions on Dependable and Secure Computing, vol. 3, no.1

[16] O. Younis and S. Fahmy, (2004)"HEED: A Hybrid, Energy- Efficient Distributed Clustering Approach for Ad Hoc Sensor Networks," IEEETransactions Mobile Computing, vol. 3, no. 4, pp. 366-379, December.

[17] Ali Sadollah,' water cycle algorithm: a detail standard code DOI: 10.1016/j.softx.2016.03.00

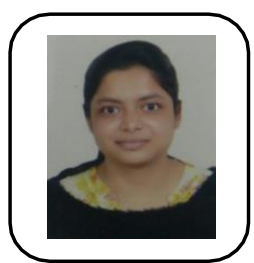

\section{AUTHORS PROFILE}

Shradha Yadav, final year $M$ tech student in (Electronics and communication) in Chandigarh engineering college,

landran.CurrentlydoingresearchworkinWirelesssensorsNetwork.Attendedmulti pleworkshopregardingwirelesssensorsnetwork. Very enthusiastic person and constantly want to improve and learn new thing which help in my research work .Received degree in BTECH (Electronics and communication) from Punjab Technical University. Have made multiple projects including hardware and software and attended seminars during graduation.

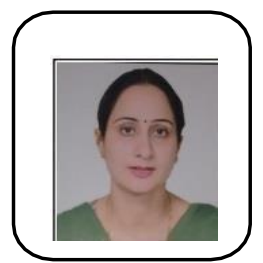

Shalley Bakshi, BE (ECE) from Pune university and mtech from PTU. A dedicated and enthusiastic educator with about 14 years of experience with a passionate commitment towards student development and have the right blend of teaching experience and industry knowledge .Regularly attending well in (telecom field) renounced national conferences and internationals journals. seminars. Published 11 papers in various nationals and

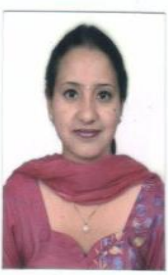

Manpreet Kaur, assistant professors in Chandigarh engineering college, landran .Department electronic and communication. Having more than 5 years of teaching experience. Very passionate and commitment towards student development. Regularly attending various seminars and conferences regarding wireless communication and published various papers in nationals and internationals journals. 